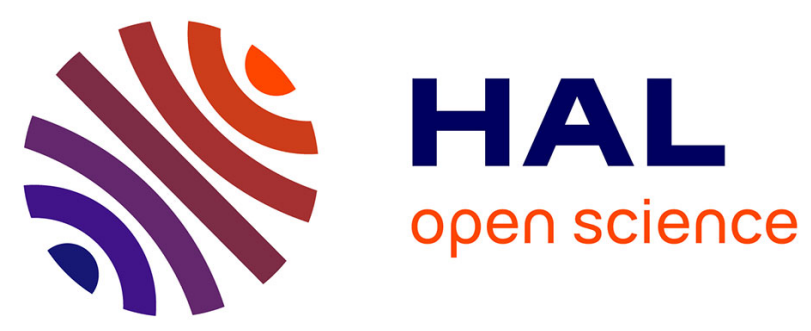

\title{
A cross-layer MAC and routing protocol based on slotted Aloha for Wireless Sensor Networks
}

David Espes, Xavier Lagrange, Luis Alberto Suarez Rivera

\section{To cite this version:}

David Espes, Xavier Lagrange, Luis Alberto Suarez Rivera. A cross-layer MAC and routing protocol based on slotted Aloha for Wireless Sensor Networks. Annals of Telecommunications - annales des télécommunications, 2015, 70 (3-4), pp.ISSN 0003-4347. 10.1007/s12243-014-0433-8 . hal-01009637

\section{HAL Id: hal-01009637 \\ https://hal.science/hal-01009637}

Submitted on 18 Jun 2014

HAL is a multi-disciplinary open access archive for the deposit and dissemination of scientific research documents, whether they are published or not. The documents may come from teaching and research institutions in France or abroad, or from public or private research centers.
L'archive ouverte pluridisciplinaire HAL, est destinée au dépôt et à la diffusion de documents scientifiques de niveau recherche, publiés ou non, émanant des établissements d'enseignement et de recherche français ou étrangers, des laboratoires publics ou privés. 


\title{
A cross-layer MAC and routing protocol based on slotted Aloha for Wireless Sensor Networks*
}

\author{
David Espes ${ }^{\dagger}$ \\ LabSTICC, University of Brest, Brest, France \\ Xavier Lagrange and Luis Suárez \\ IRISA, RSM Department, Telecom-Bretagne, Rennes, France
}

Dated: April 22, 2013

\begin{abstract}
Wireless Sensor Networks (WSN) consist of a large number of sensors which have limited battery power. One of the major issues in WSN is the need to improve the overall network lifetime. Hence, WSN necessitate energy-efficient routing protocols. In this paper, a crosslayer routing protocol (PLOSA) is designed to offer a high delivery rate, a low end-to-end delay and a low energy consumption. To achieve these goals, the transmission channel is divided into different slots and a sensor has access to a slot related to its distance from the collector. The transmissions are then ordered within the frame from the farthest nodes to the closest ones which is a key point in order to ease forwarding and to conserve energy. We have conducted simulation-based evaluations to compare the performance of the proposed protocol against the framed aloha protocol. The performance results show that our protocol is a good candidate for WSN.
\end{abstract}

Keywords: sensor networks, energy-awareness, cross-layer protocol, medium access control, routing

\section{Introduction}

Wireless sensor networks (WSN) are used in a wide range of applications as military, health and transport. Sensors have limited battery power. In most applications, they are required to be operating in the order of months to years. Generally, these batteries cannot be replaced because sensors are deployed in specific areas with no maintenance. Hence, sensors can only transmit a finite number of packets before exhausting their battery power. Multi-hop networking is then necessary for a data packet generated by a sensor to be able to reach its final destination with a limited transmission power [1]. Furthermore, a common mechanism to reduce energy consumption is to turn the transceiver of sensor nodes into a low power sleep state when it is not being used.

\footnotetext{
*The final publication is available at Springer via http://dx.doi.org/10.1007/s12243-014-0433-8

${ }^{\dagger}$ Electronic address: david.espes@univ-brest.fr; Corresponding author
} 
Unlike traditional networks, WSN have their own design and resource constraints. The design constraints are application dependant and are based on monitored environment [2]. Whatever the design approach, it is essential that WSN are subject to a rigorous analysis to provide longterm survivability of the architecture. The OSI (Open Systems Interconnection) layer model is generally used to specify the protocol architecture. However, due to the lack of memory and energy, it becomes difficult to use the traditional layer model in WSN [3]. Cross-layer design is proposed to achieve gains in overall system performance in wireless networks.

Cross-layer techniques improve energy conservation in WSN [4, 5]. Hence, most cross-layer routing protocols have been proposed to reduce energy consumption in WSN [6-10]. These routing protocols are efficient solutions for energy conservation. They use MAC (Media Access Control) layer information such as joint scheduling, power control, and sleep state of sensor nodes, to control energy consumption.

In [6], the authors propose a cross-layer approach to compute the minimum transmission power level between nodes and find a route between the nodes and the collector. Getting the proper transmission power level reduces the power consumption and decreases the interference between nodes. Each node adjusts its transmission power before sending a data packet. When a node receives one, it sends back a message including the Received Signal Strength Indicator (RSSI) value. Each node maintains a table with the proper transmission power according to the RSSI value. The nodes compute a routing algorithm that uses this value to find a route with the collector.

In [7], a cross-layer protocol integrates MAC and routing functionalities to support geographic forwarding. It is assumed that the destination location is known. This protocol adjusts the transmission power level in order to reduce the energy consumption. Nodes select the best next relay node while forwarding packets to the destination. To this aim, the nodes use a weighted factor representing the progress toward the destination per unit of transmission power.

In [8], a cross-layer protocol combines an adaptive synchronous MAC scheme and a tree-based energy aware routing algorithm to achieve the reduction in energy consumption. If there are no data to send or receive, the node turns off its transceiver to reduce the time and energy wasted in idle listening. The routing algorithm uses two metrics on a link (the link error rate and the energy cost) to find a tree path to the collector. A path with many short-range links reduces the energy consumption due to the nodes transmission power adjustment. In some cases, this type of path can cause more link errors that result in more retransmissions. To combine these two parameters enhances the energy consumption in the network.

In [9], researchers propose a cross-layer architecture using MAC and routing layer. This crosslayer protocol extends the 802.11 MAC protocol [11] and the Dynamic Source Routing (DSR) protocol [12]. DSR is not featured to determine whether a packet loss is due to congestion or node failure. When the DSR protocol detects a loss, it reinitiates a new path discovery increasing the overhead. If the communication breaks because of congestion, this leads to inefficient energy utilization. To overcome this problem, the authors propose that the nodes keep a record of the last received power level from each neighbor nodes. Hence a node can determine whether the neighboring node remains within the transmission range. This information is passed to the DSR protocol which determines if the loss occurs because of congestion. In this case the path discovery is not initiated.

In [10], the authors propose a geographic cross-layer routing protocol that does not require location awareness to forward packets. The collector sends a beacon frame periodically. When a sensor receives one, it measures the RSSI value. When a node has some data to transmit, it adds its RSSI value to the header of the Request to Send (RTS) frame and broadcasts it. Only nodes that are closer to the collector (i.e. they have a higher RSSI value than that of the sender) 
can participate at the contention mechanism. These nodes choose random time slots within a contention window size. The node that has the earliest timeout sends a Clear to Send (CTS) frame and is determined as the next hop node.

Due to the large diversity of applications, WSN can be classified on the basis of hardware and application requirements [13]. In a lot of cases, WSN are composed of heterogeneous sensors (deployed over a physical area of interest) to sense environmental data and deliver them to a collector and then to an end-application. This type of WSN is called Wireless Data Collection Networks (WDCN). It enables applications to observe the variation of a particular physical signal during a period of time.

In this paper, we use the cross-layer approach to design a new protocol, PLOSA (Path-loss Ordered Slotted Aloha protocol), for WDCN. PLOSA modifies frame aloha to reduce energy consumption. The frame aloha protocol is a widely used access protocol that is characterized by its simplicity, establishing itself as a good candidate for WDCN. However the price of its simplicity is a lack of fairness in media access. Nodes are at various distances from the collector. In free space propagation model, signal attenuation is strictly related to the distance between the transmitter and the receiver. The received signal strength of distant nodes is significantly lower than those of close nodes. Due to the capture effect, distant nodes have a lower throughput than close nodes. In WSN, the utilization of a multi-hop mechanism avoids the capture effect. PLOSA proposes a multi-hop cross-layer routing protocol where the idea is to order the access of nodes to optimize the energy consumption. The transmission channel is divided into different slots and a node has access to a slot related to its distance. The higher the distance between a node and the collector, the earlier this one can access a slot. Once the access of nodes is ordered, the resulting routing protocol is very simple. Indeed it does not require the notion of routing table (the next forwarding hop is always closer to the collector). Our protocol reduces at a minimum the overhead in both the routing protocol and the collision avoidance mechanism. No routing information are required to find a path between a sensor and the collector. Each time a node sends a packet, a closer one to the collector forwards it until it reaches the collector. In the same way, the number of collisions is limited as the access of nodes is ordered. A collision can only occur in the vicinity of a sender node i.e. two nodes can send a packet into the same time slot if they are at the same distance from the collector. Hence our protocol avoids the hidden node problem without the use of an intrusive collision avoidance mechanism as RTS/CTS handshake. To our knowledge, no other cross-layer routing protocol exists addressing the question of how avoiding routing overhead and hidden node problem. Indeed PLOSA protocol is designed to offer high delivery rate and low end-to-end delay. In most cases PLOSA provides data delivery to the collector within one frame.

The rest of this paper is organized as follows. In Section 2, PLOSA protocol is described in details. In Section 3, the performance of this protocol is discussed and compared to the frame aloha protocol. Finally, we make some concluding remarks in Section 4.

\section{Protocol Description}

\subsection{Background and Assumptions}

We consider a wireless data collection network model with a large number of sensors and one collector. Data are generated by the sensors and put into packets that are transmitted to the collector by use of a multi-hop forwarding if necessary. Each sensor has a unique identifier that is appended to the information field in the packet to identify the source of the data. Each packet has also a unique identifier called PACKET_ID. As the forwarding process can generate 
duplications, PACKET_ID can also be used if detecting duplicated packets is required by the application.

The transmission power of the collector is assumed to be high enough to reach all sensors in the network. As the collector is generally connected to the mains power source, it is not a restrictive assumption. Furthermore, if there are some limitation on the collector maximum power, spread spectrum techniques can be used to have a large transmission range with a moderate transmission power.

The collector regularly transmits a beacon packet that includes the used transmission power. All sensors receive the packet and measure the received power level. Several measurement samples may be used to calculate an average received level in order to mitigate the multipath fading. The difference between the transmission power and the received power in $\mathrm{dB}$ is then the path loss between the node and the collector.

A basic assumption of the protocol is that the path loss is an increasing function of the distance. Because of the shadowing effect, this is not strictly true but is valid for outdoor environment, which represents a large panel of applications.

As stated before, the access mechanism is based on frame slotted aloha. After each beacon packet, a frame made of $S$ slots (numbered from 0 to $S-1$ ) is then defined. A packet is always transmitted within a slot.

The proposed access mechanism can be used for different environments. However for the sake of clarity, all examples will be given for a typical outdoor propagation: the path loss between two nodes is $L=r^{\alpha} 10^{\xi / 10} / k$ where $r$ is the distance between both nodes, $k$ and $\alpha$ are environmentdependant parameters $(\alpha \in[2,4]$ and is 2 for free space propagation and typically 3.5 for outdoor rural or indoor environment), $\xi$ is a random Gaussian variable that models the shadowing effect.

The PLOSA protocol is a Cross-Layer protocol. The network layer uses information of the link layer to access the medium efficiently. The forwarding process is composed of four steps: the listening window, the forwarder selection, the transmission window and the acknowledgement window. For the sake of clarity, we first present the transmission window that introduces the core of the protocol and the main parameters.

\subsection{Transmission window}

\subsubsection{Main principle}

Let $N$ be the number of sensors and $L_{i}$ be the path loss between the sensor $i$ and the collector (in linear scale). We assume the system is designed for a maximum path loss denoted as $L_{\max }$. Let $y=f(x)$ be a decreasing function that goes from $[0,1]$ to $[0,1]$. Function $f$ defines the access characteristics and is the core function of the process.

Let us assume Node $i$ has a packet to transmit. It can be either a piece of information generated by sensor $i$ or a packet sent by another node that has to be forwarded. Node $i$ computes $s_{i}=\left\lfloor S f\left(L_{i} / L_{\max }\right)\right\rfloor$. Node $i$ uses slot $s_{i}$ as reference slot for transmission. As $f$ is a decreasing function, slot $s_{i}$ is at the beginning of the frame if node $i$ is far from the collector and at the end of the frame if node $i$ is close to the collector. Transmissions are then ordered within the frame from the farthest nodes to the closest ones, which is a key point to ease forwarding and to conserve energy in that process.

Function $f$ must be carefully chosen in order to optimize the access mechanism. A first objective is to equally spread the packets on all slots of the frame. If nodes are uniformly distributed in a disk of radius $\mathrm{R}$ centered on the collector, the cumulative distribution function (cdf) of radius $r$ without shadowing is $1-(r / R)^{2}$. The cdf of the path loss is then $1-\left(L / L_{\max }\right)^{2 / \alpha}$ as $L_{\max }=R^{\alpha} / k$. In a 1-hop system without forwarding, function $f$ must 


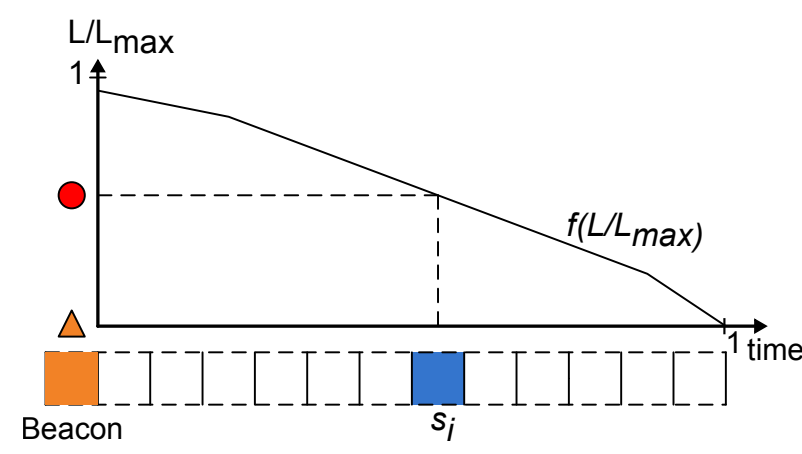

a)

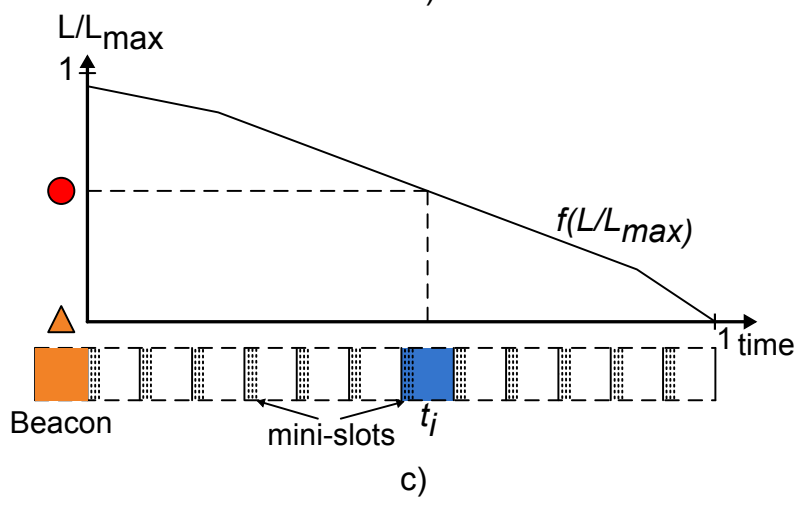

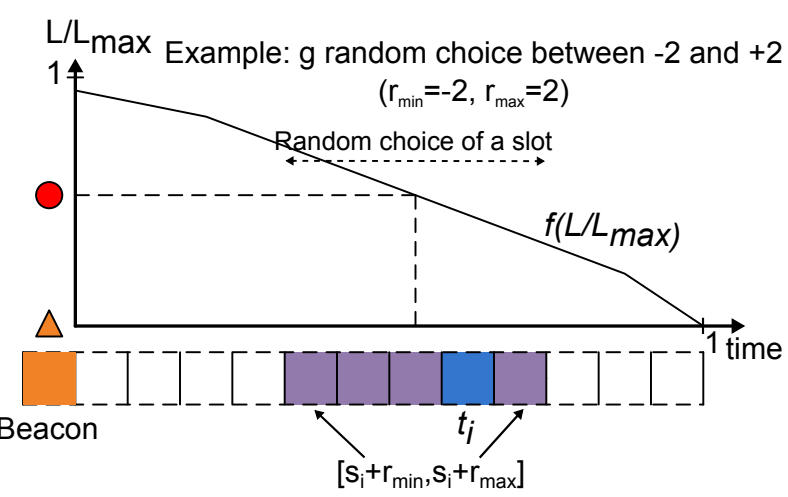

b)

Legend:

node i

$\triangle \operatorname{sink}$

Figure 1: Transmission window mechanisms. a) Main principle. b) Random slot selection. c) Mini-slot selection. 
be chosen as $f\left(L_{i} / L_{\max }\right)=1-\left(L_{i} / L_{\max }\right)^{2 / \alpha}$ in order to have the same probability for any slot to be used. In case of forwarding it can be shown that function $f$ must be

$$
f\left(\frac{L_{i}}{L_{\max }}\right)=1-\left(\frac{L_{i}}{L_{\max }}\right)^{1 / \alpha}
$$

By definition, slot $s_{i}$ is always between 0 and $S-1$ (see figure 1.a). When two or more nodes transmit a packet in the same slot a collision occurs and nodes retransmit the packets in the next frame. If the reference slot is the same between two frames, nodes retransmit the packets in the same slot and create a new collision. In such a case, repeated collisions waste the throughput of the network and increase energy consumption. To reduce this type of collision we propose two mechanisms to reduce the probability that a node accesses the same slot in two successive frames.

\subsubsection{Random slot selection}

In order to avoid repeated contentions a random process is introduced. The random process extends the transmission window length i.e. the transmission slot is chosen to be into a part of the frame centered on the reference slot. This process reduces the probability to have two consecutive transmissions by the same node into the same slot. Let $r$ be a discrete random variable with integer values and $g$ be its probability mass function (function $g$ is defined on $\mathbb{Z}$ ). Node $i$ then draws a value $r_{i}$ for $r$ and transmits a packet in slot

$$
t_{i}=\max \left(0, \min \left(s_{i}+r_{i}, S-1\right)\right)
$$

Let $r_{\min }$ and $r_{\max }$ be respectively the lowest and highest values of random variable $r$ (note that $\left.r_{\min } \leq 0\right)$. All possible slots for transmission are then contained in $\left[s_{i}+r_{\min }, s_{i}+r_{\max }\right]$ (see figure 1.b).

\subsubsection{Mini-slot selection}

Another method to reduce repeated collisions is to use mini-slots. In such a case each slot begins with a series of mini-slots, each of which has a duration equal to the maximum propagation delay. The duration of a time slot is equal to the data transmission time plus some number of mini-slots time. Before sending a packet each node $i$ computes its transmission slot $t_{i}=s_{i}$ and chooses a mini-slot randomly (see figure 1.c). At the beginning of its mini-slot a node sends a packet only if the channel is sensed idle. The probability of having a collision is reduced according to the number of mini-slots. Let us assume the mini-slot selection method follows a uniform distribution. Let $M$ be the number of mini-slots. Let $N$ be the nodes that access to the same slot. If we assume all nodes can listen to each other, no collision occurs if the first chosen mini-slot is selected by only one node i.e. the probability $P_{c}$ to have a collision is $P_{c}=1-\frac{N}{M} \sum_{i=1}^{M-1}((M-i) / M)^{N-1}$. We refer to this version of our protocol as PLOSA_MS.

\subsection{Acknowledgement process}

There is no dedicated acknowledgement message but the packet identification mechanism and the forwarding process are used as an acknowledgement process. It then occurs immediately after a packet is transmitted by a node. The node waits for at most $W_{A}$ slots (called acknowledgement window). If it receives a packet with the same PACKET_ID, then the packet is successfully forwarded and the node can go to a sleep mode. If the window expires, the packet is transmitted again in the next frame. See figure 2. 


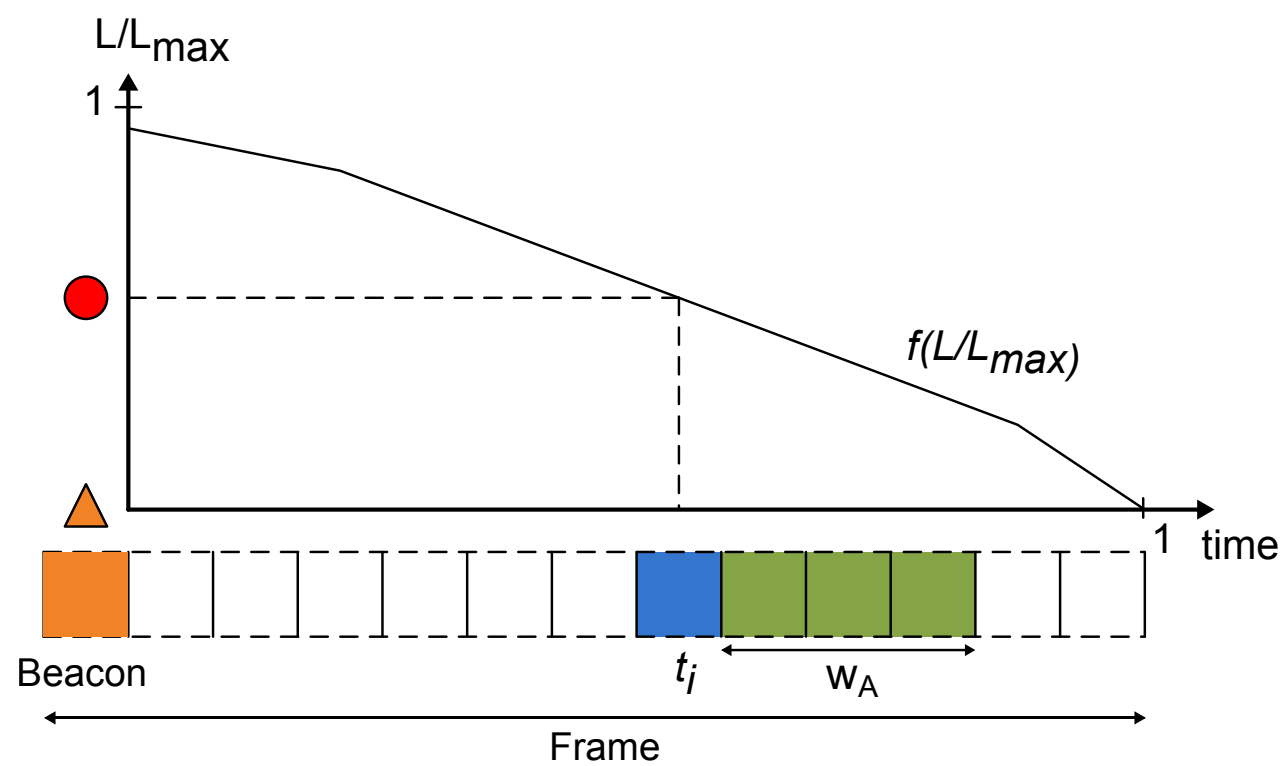

Figure 2: Acknowledgement Window

All packets received by the collector are acknowledged in the beacon packet of the following frame. The beacon packet can then be used both an acknowledgement on the last hop and an end-to-end acknowledgement.

\subsection{Listening window}

Each node may need to forward packets from nodes farther from the collector and then has to listen to the transmission channel for a given duration. This duration must be large enough to enable an efficient relaying process but must also be as low as possible to conserve energy. Let us consider node $i$ that is looking for possible packets to be forwarded. As transmissions are ordered, there is no need to listen to slots after slot $s_{i}+r_{i}$, which is the chosen slot for a possible transmission in the frame. Furthermore, slot numbers which are very low compared to $s_{i}$ are used by nodes very far from node $i$. Node $i$ has then to listen to slot numbers lower than but close to $s_{i}$. We then define two parameters $\delta$ and $W$, which are positive integers: node $i$ listens to slot numbers between $s_{i}-\delta-W$ and $s_{i}-\delta$. If no packet is received, node $i$ enters a low consumption state (sleeping) from slot $s_{i}-\delta$ till the end of the frame. If a packet is received, node $i$ may then be a forwarding candidate and uses the forwarder selection process (see section 2.5).

In order to have a listening window and transmission window without a gap in between, $\delta$ may be chosen as $\delta=-r_{\min }+1$. Note that $W$ determines the maximum width of the listening window. See figure 3 .

\subsection{Forwarder selection}

The forwarder selection is used to find a node in order to be the next-hop forwarder for a given packet. The node that forwards the packet is then called the forwarder. All data packets contain the path loss value between the sender and the collector within the header. Let us consider node $j$, which receives a packet sent by node $i$. If $L_{i} \leq L_{j}$ then node $j$ has a larger path loss compared to $i$ and therefore cannot be a candidate (due to the path-loss ordered listening window selection, 


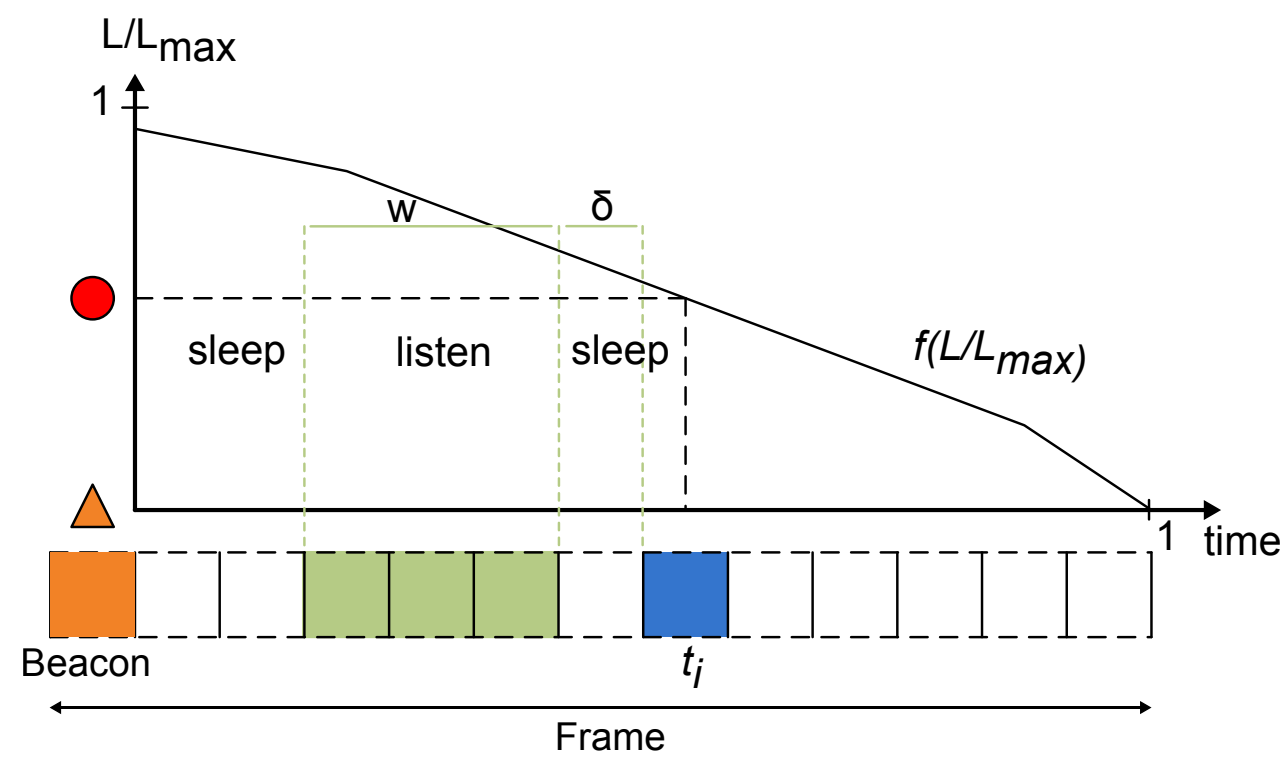

Figure 3: Listening Window

this event occurs with a low probability). If node $L_{i}>L_{j}$ then node $j$ is a candidate.

For a same packet to be forwarded, it is possible to have more than one forwarding candidate. The way to decide who is going to be the forwarder is solved by letting the nodes prepare for transmissions (see section 2.2). The first one that transmits is the forwarder. Each packet includes a unique packet identifier. As soon as a forwarder candidate is correctly receiving a packet with the same identity than the one for which it is candidate it then leaves out the forwarder selection process and enters a low consumption state till the end of the frame.

\section{Performance of the Protocol}

\subsection{Presentation of the reference protocol}

In order to evaluate the performance of PLOSA, we compare it with a simple one-hop slotted aloha access. Spread spectrum is used to improve the transmission range of sensors and to allow them to reach the collector (see 3.2 for the considered value). Let $S F$ be the spreading factor. The packet transmission time is then multiplied by $S F$ compared to non-spread multihop transmission. In order to keep the same frame period, the number of slots in the frame is divided by $S F$. The access mechanism is a standard frame Aloha. When a node has a packet to transmit, it randomly chooses a slot in the frame and transmits the packet. If the packet is acknowledged by the collector at the beginning of the next frame, the node leaves the process. In other cases, the packet is retransmitted until acknowledgement by the collector or the maximum number of transmissions is reached.

\subsection{System Parameters}

Main radio parameters like the transmission power, the noise factor, the data rate are the same as for IEEE 802.15.4. We just consider a higher transmission power $(100 \mathrm{~mW})$ for the collector. Sensitivity and power consumption are in accordance with Tmote devices [14].

According to the transmission powers and the sensitivity, a one-hop transmission requires a 
20-dB spreading gain. As spreading factors are generally powers of 2 we assume the spreading factor is 64 .

The pathloss is assumed to be $r^{\alpha} / k$ with $\alpha=3, k=3.16210^{-6}$ and $r$ given in meters. In $\mathrm{dB}$ the pathloss is then $55+30 \log _{10}(r)$ as in [15]. It corresponds to a typical outdoor or open-space indoor propagation. The transmission range of a node is then 20 meters whereas the transmission range of the collector is 93 meters.

The simulated network is composed of 160 nodes. The network size is a disk of radius 100 meters. To provide much more realistic scenarios than free space propagation, we consider random topology simulations with shadowing effect. The shadowing is represented as a lognormal random variable with standard deviation $\sigma$ of $3.8 \mathrm{~dB}$ (and $0 \mathrm{~dB}$ average).

A 4-state model is used for power consumption. In sleep-mode the consumed power is assumed to be low enough to be neglected. In idle mode, the radio module is on and the consumed power is $10 \mathrm{~mW}$. In reception mode (when a packet is currently decoded) signal processing increases the required energy and the consumed power is $60 \mathrm{~mW}$. For a 1-mW transmission, the consumed power is $52 \mathrm{~mW}$. Once again, such figures are in accordance with [14].

For the simulations of our protocol, the OPNET discrete event simulator is used. We compare our protocols to the framed Aloha protocol (cf. section 3.1). Sensors are considered static, as is usual in certain application scenarios. In the simulation, the collector node is located in the center of the network. At the beginning of each frame (frame duration=83.86 ms) it sends a beacon packet of 160 bits. The frame is composed of 64 data slots for a multi-hop process ( 1 for the slotted aloha). The time slot duration available for a data transmission and a beacon transmission is respectively $1.3 \mathrm{~ms}$ and $0.66 \mathrm{~ms}$. The number of mini-slots is 8 , each of which has a duration of $2 \mu \mathrm{s}$. New packets $(360 \mathrm{bits})$ are generated according to a Poisson process in each sensor. Independent processes are considered between nodes. The simulation runs for 1000 seconds.

\subsection{Simulation Results}

The access mechanism is analyzed in term of network bandwidth utilization, delivery delay to the collector and consumed energy for various load. The load is expressed as the average number of new packets per slot. It can be easily expressed as a function of $\lambda$, the average number of new packets per time for a node. Let $N$ be the number of nodes in the system and $T_{\text {frame }}$ the duration of the frame. The offered load is given by $N \lambda T_{\text {frame }} / S$. Due to the spreading factor the slots of the framed Aloha process is 64 times larger than those in our protocols. In the results the offered load is expressed for our protocols. The offered load for the framed Aloha protocol is $64 N \lambda T_{\text {frame }} / S$. In other words, the same load for PLOSA and Aloha corresponds to the same new packet rate (same $\lambda$ ).

Figure 4 highlights the packet loss rate under different densities. The packet loss rate is the ratio of the number of packets that are not received by the collector to the number of packets being generated at the source nodes. The results show that our protocols outperform the framed Aloha protocol because our protocols have fewer packet losses than the framed Aloha protocol. The network bandwidth is used at its utmost and our protocols are really designed to treat more flows or the same number of flows but with more bandwidth. When the offered load is low, the packet loss rate returned by the framed Aloha protocol is 50 times as much as the one returned by our protocols. Due to the spreading factor, the sensors at the edge of the networks have a higher PER when the framed aloha protocol is used. Moreover the time to transmit a packet is 64 as high with the framed Aloha protocol as with our protocols. In such a case the hidden node effect is more pronounced and increases the probability of having a collision. These conditions 


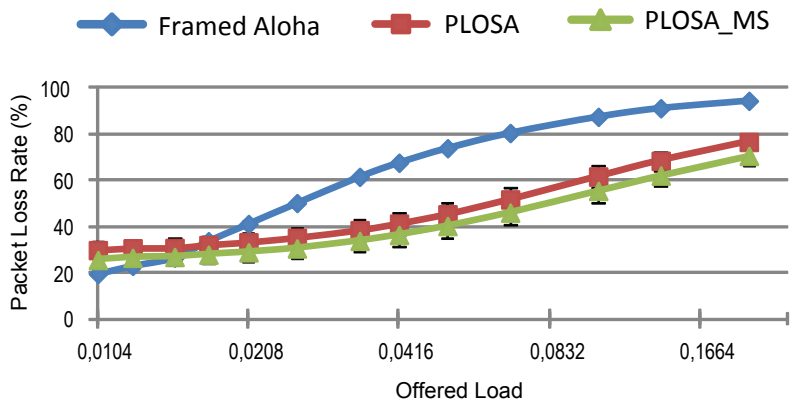

a)

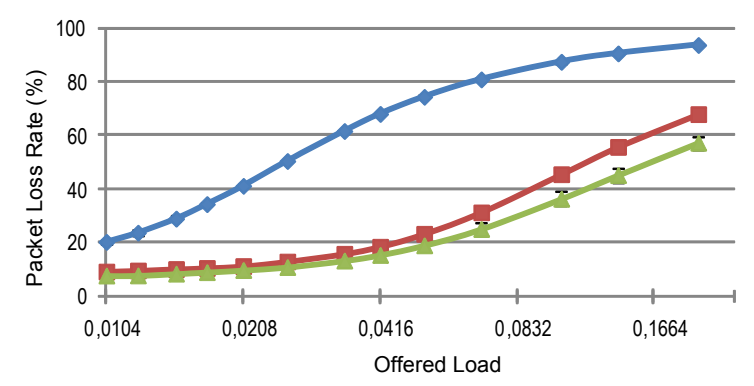

b)

Figure 4: Packet Loss Rate under different topologies and densities. a) 80-node random topology. b) 160-node random topology.

increase the packet loss rate of the framed Aloha protocol. At high load, our protocols are nearly $65 \%$ as efficient as the framed Aloha protocol because the packet emission is regulated in the frame in order to reduce the packet loss rate. Collisions may only occur when nodes are located at the same distance of the collector and in the same vicinity but our protocols avoid the hidden nodes effect by using the network bandwidth efficiently. The PLOSA_MS protocol has lower packet loss rate than the PLOSA protocol thanks to the use of mini-slots that prevent two nodes from transmitting simultaneously.

Figure 5 and Figure 6 show the energy consumption of the protocols. Our protocols limit the increase in energy consumption thanks to a low packet loss rate. Our sensors do not need any extra time to listen to the medium. A low energy level is an important criteria to extend the network's lifetime. When the offered load is low the frame Aloha performs better from an energy point of view. Sensors using the framed Aloha protocol do not listen to the medium except to receive the beacons. On the other hand, each node using our protocols listens to the medium in order to receive packets or to acknowledge transmitted packets. When the load grows the trend is reversed. A total energy reduction of $55 \%$ can be obtained by our protocols. The PLOSA_MS has lower energy consumption than the PLOSA protocol due to the use of mini-slots. A node senses the medium during the mini-slots. If another node sends a packet, it delays its transmission and enters sleeping mode. Nodes stay longer in sleep mode than nodes using the PLOSA protocol. The longer nodes stay in the sleeping mode, the lower the energy consumption.

The simulations (Figure 7 and 8) show that the delay increases slightly to match the increase of the offered load. Our protocols are better when the load is low whatever the network topology. The spreading factor increases the range, however it reduces the bandwidth. For a one-hop process, the time to send a packet is the same whatever the position of a node in the network. The transmission time is equal to the frame duration. For a multihop process the time is related to the distance between a node and the collector. Hence our protocols have a lower end-to-end delay. At high load the framed Aloha protocol has a lower end-to-end delay than our protocols. The number of retransmissions increases with the traffic load. It is limited to 3 per node. In the worst case a packet is retransmitted three times with a one-hop mechanism whereas it is retransmitted nine times with a multihop process of three hops (average hop count). Hence a multihop process degrades the end-to-end delay slightly. In order to reduce this effect, our protocols are well-designed to decrease the packet loss rate. For all protocols, the end-to-end delay does not exceed 0.6225 seconds which is quite acceptable to transmit QoS traffic. 


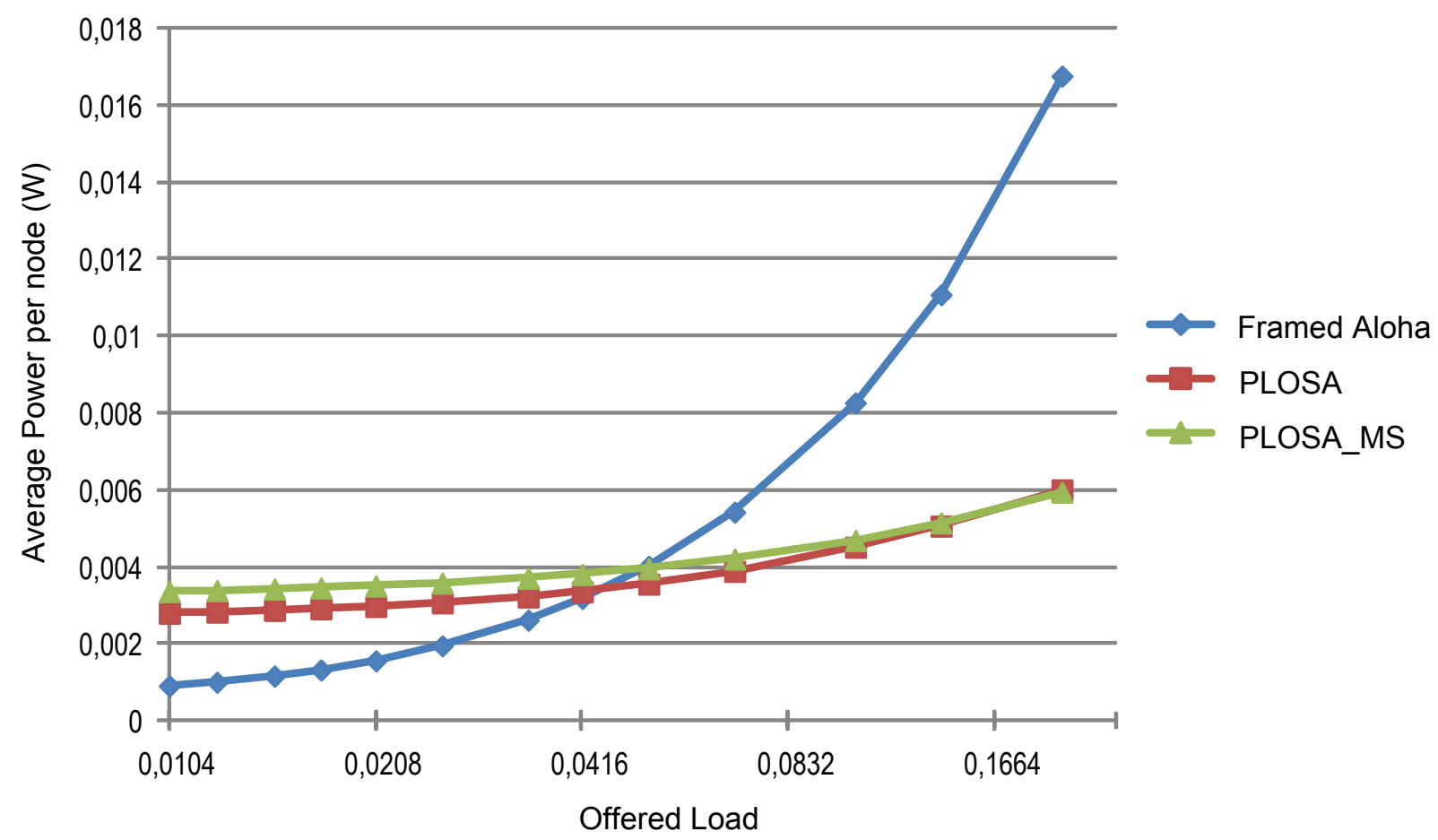

Figure 5: Average power per node.

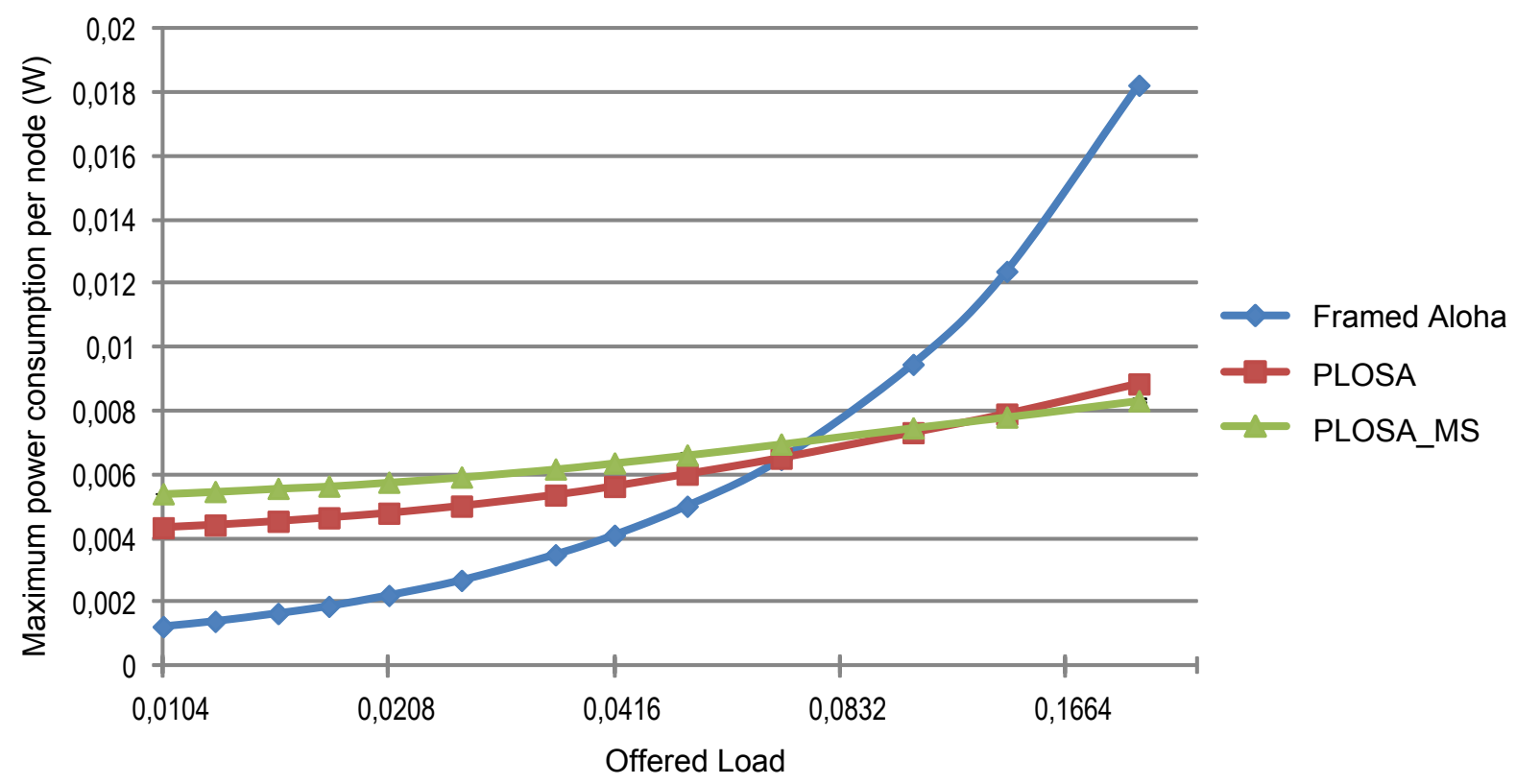

Figure 6: Maximum energy consumption. 


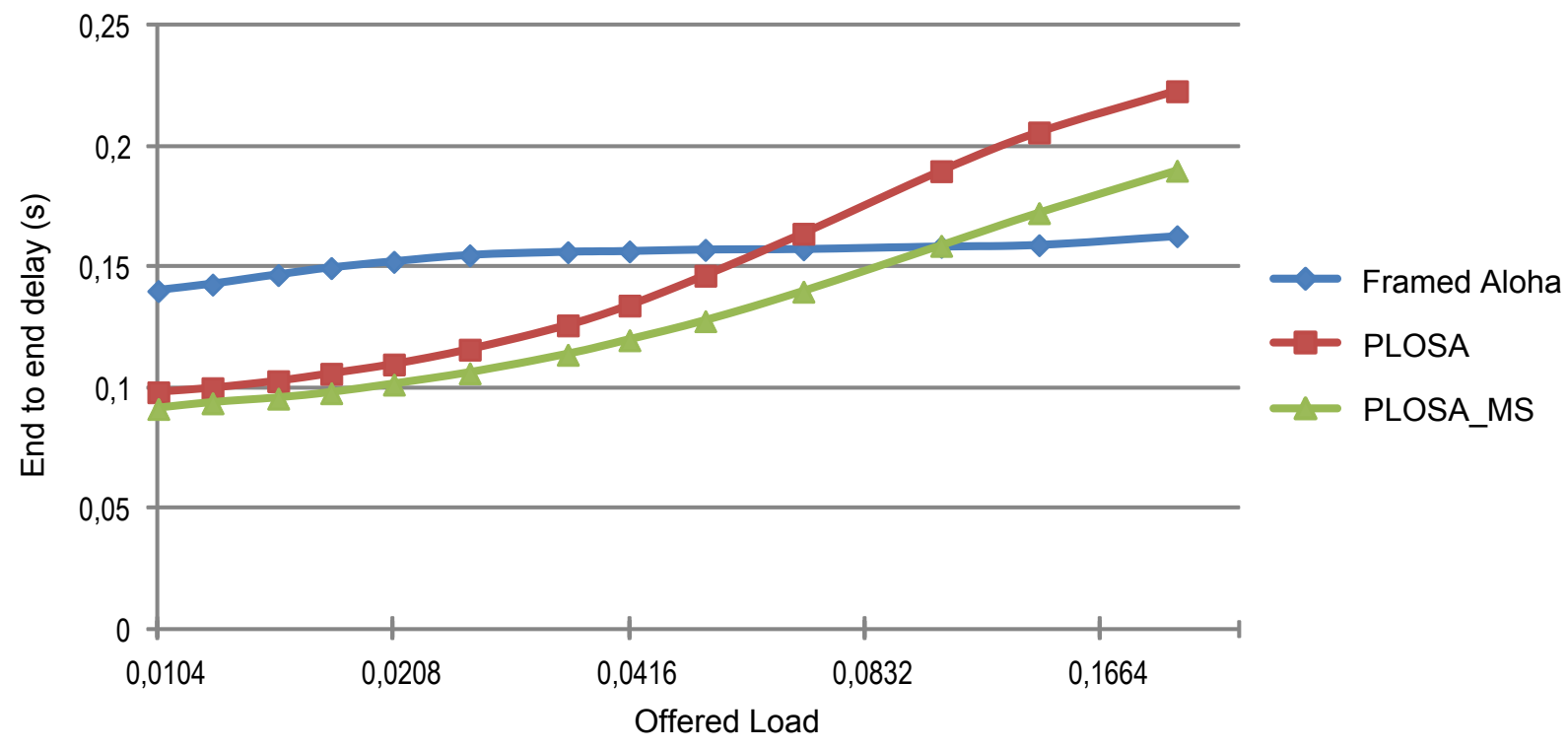

Figure 7: Average End-to-End delay.

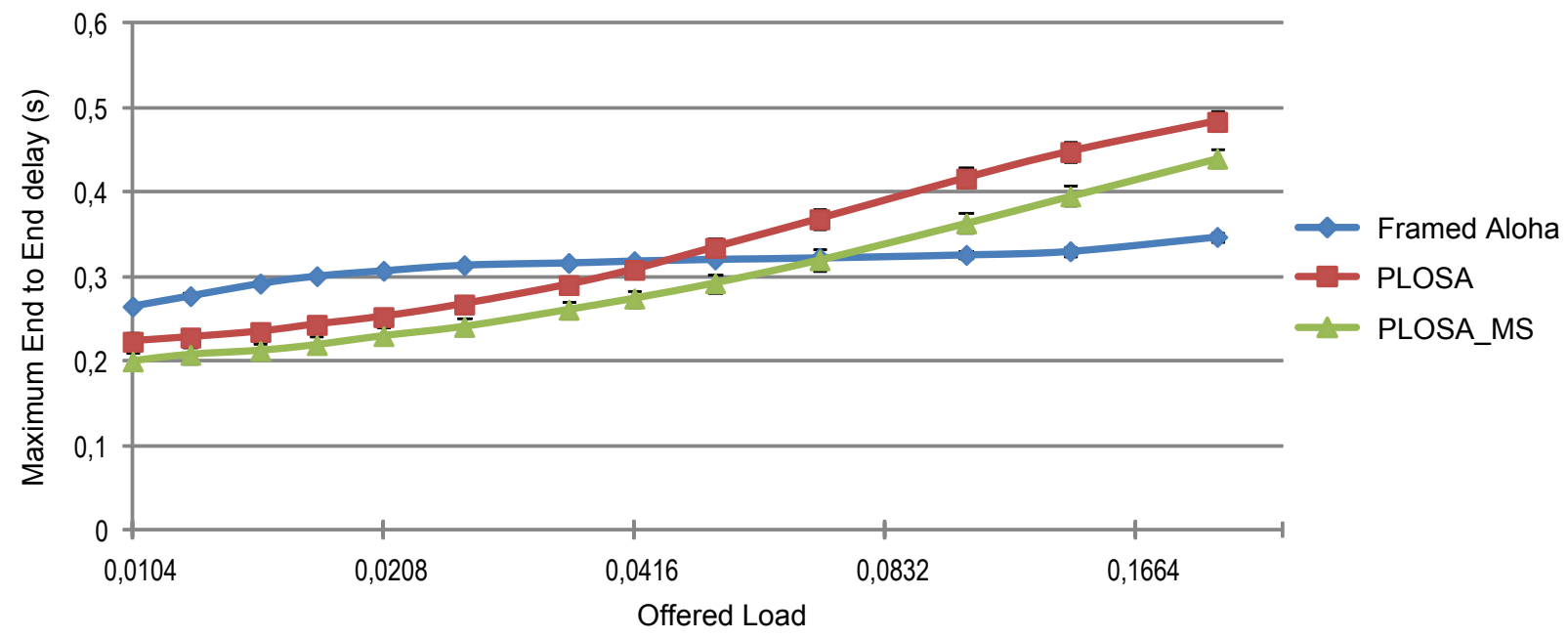

Figure 8: Maximum End-to-End delay. 


\section{Conclusion}

Due to the limited storage, energy, and computational resources of WSN, the MAC or routing techniques developed for other types of network are not adequate for them. The solution proposed here for reducing energy consumption uses a cross-layer method where communication between nonadjacent layers is enabled.

The multi-hop access mechanism we propose in this paper distributes the node access in the frame according to their distance to the collector. The forwarding process is then simplified and can be done within a frame. Furthermore, it is possible to optimize sleeping periods of devices because each node can receive packets to be forwarded only in a specific part of the frame. PLOSA and PLOSA_MS were studied for networks with fixed nodes. However as the routing process is stateless, it can easily be used for mobile networks. Generalizing PLOSA for ad-hoc networks is then a possible extension of this work.

\section{References}

[1] I.F. Akyildiz, W. Su, Y. Sankarasubramanian, and E. Cayirci. A survey on sensor networks. IEEE Communications Magazine, 40:102-114, 2002.

[2] J. Yick, B. Mukherjee, and D. Ghosal. Wireless sensor network survey. Computer Networks, 52:2292-2330, 2008.

[3] A.J. Goldsmith and S.B. Wicker. Design challenges for energy-constrained ad hoc wireless networks. IEEE Wireless Communications, 9(4):8-27, 2002.

[4] L. van Hoesel, T. Nieberg, J. Wu, and P.J.M. Havinga. Prolonging the lifetime of wireless sensor networks by cross-layer interaction. IEEE Wireless Communications, 11(6):78-86, 2004 .

[5] W. Su and T.L. Lim. Cross-layer design and optimization for wireless sensor networks. Software Engineering, Artificial Intelligence, Networking, and Parallel/Distributed Computing, pages $278-284,2006$.

[6] Y. Bai, S. Liu, M. Sha, Y. Lu, and C. Xu. An energy optimization protocol based on cross-layer for wireless sensor networks. Journal of Communications, 3(6):27-34, 2008.

[7] L. Galluccio, A. Leonardi, G. Morabito, and S. Palazzo. A mac/routing cross-layer approach to geographic forwarding in wireless sensor networks. Ad Hoc Networks, 5(6):872-884, 2007.

[8] J. Kim, J. Lee, and S. Kim. An enhanced cross-layer protocol for energy efficiency in wireless sensor networks. Proceedings of the 2009 Third International Conference on Sensor Technologies and Applications, pages 657-664, 2009.

[9] N. Chilamkurti, S. Zeadally, A. Vasilakos, and V. Sharma. Cross-layer support for energy efficient routing in wireless sensor networks. Journal of Sensors, 2009.

[10] A. Awang, X. Lagrange, and D.R. Sanchez. Rssi-based forwarding for multihop wireless sensor networks. Lecture Notes in Computer Science, 5733:138-147, August 2009.

[11] IEEE Standard for Information Technology. Local and Metropolitan Area Network Specific Requirements Part 11: Wireless LAN Medium Access Control (MAC) and Physical Layer 
(PHY) Specifications Further Higher-Speed Physical Layer Extension in the 2.4 GHz Band. 2003.

[12] D. Johnson, Y. Hu, and D. Maltz. The dynamic source routing protocol (dsr) for mobile ad hoc networks for ipv4. RFC 4728, 2007.

[13] I. Mabrouki, G. Froc, and X. Lagrange. Architectural classification for the design of wireless sensor networks. Sème Journées Doctorales en Informatique et Réseaux (FDIR'08), 2007.

[14] Moteiv Corporation. Ultra low power IEEE 802.15.4 compliant wireless sensor module. Datasheet specification, 2006.

[15] M.C. Vuran and I.F. Akyildiz. Cross-layer analysis of error control in wireless sensor networks. IEEE International Conferance on Sensor and Ad-hoc Communications and Networks, 2:585-594, 2006. 